\title{
Magnetic resonance imaging to detect vesico-symphyseal fistula following robotic prostatectomy
}

\author{
Ryan C. Hutchinson, David D. Thiel, Joseph M. Bestic \\ Department of Urology (RCH, DDT) and Department of Radiology (JMB) Mayo Clinic, Jacksonville, \\ Florida, USA
}

\section{ABSTRACT}

Pubic complications following radical prostatectomy are rare. Osteitis pubis typically presents with symptoms related to irritation of the pubic rami including pain with ambulation and adduction of the leg. A 60-year-old male with prostatic adenocarcinoma underwent uneventful robotic assisted laparoscopic prostatectomy. The patient noted the onset of severe pubic pain exacerbated by ambulation approximately one month post-surgery. An abdominal/pelvic CT scan was negative for acute pathology. Due to continued discomfort, a multiplanar MRI of the pelvis was performed with and without intravenous contrast material ( $20 \mathrm{ml}$ Omniscan). The MRI demonstrated irregularity of the bladder base and proximal urethra with a fistulous tract extending anteriorly in direct communication with the pubic symphysis joint space. Vague periarticular marrow edema-like signal and enhancement at the pubic symphysis was thought to represent osteitis pubis. The patient's symptoms resolved after one month of urethral catheter drainage, intravenous antibiotics, and anti-inflammatory therapy.

\section{INTRODUCTION}

Abnormal connections between the symphysis pubis and the lower urinary tract with associated inflammation of the pubis are rare occurrences. First described in 1924 by Beer (1) as a sequela of simple prostatectomy, osteitis pubis typically presents with symptoms related to irritation of the pubic rami, including pain with ambulation and adduction of the leg. It is classically described as a non-suppurative process; however bacterial abscesses have been cited in association (2). More recently, this finding has been associated with transurethral resection of the prostate with similar presentation, including fever, suprapubic pain, exostoses, and bilateral adductor abscesses (2). Conservative management with urinary drainage, antibiotic therapy, and anti-inflammatory medication is standard first-line therapy for most urinary fistulae, with surgical correction reserved for unresolved cases. MRI offers excellent imaging of soft tissue-based pathology and has been used to detect urinary fistulae with good results (3).

\section{CASE REPORT}

A 60-year-old male with prostatic adenocarcinoma underwent uneventful robotic assisted laparoscopic prostatectomy. The urethral catheter was found deflated and withdrawn from the glans on the evening of post-operative day number one. The catheter was replaced uneventfully, and he was discharged home the next day. Following standard catheter removal on post-operative day nine, he was readmitted with fever, rigors, and urinary cultures positive for quinolone-sensitive E.Coli. The patient defervesced with antibiotic therapy, which was continued for 14 days.

The patient noted the onset of severe pubic pain exacerbated by ambulation approximately one month post-surgery. An abdominal/pelvic CT scan was negative for acute pathology. Continued symptomatology prompted further evaluation with dedicated pelvic MRI (Figure-1). Multiplanar MRI imaging 
of the pelvis performed with and without intravenous contrast material (20 ml Omniscan) demonstrated irregularity of the bladder base and proximal urethra with the fistulous tract extending anteriorly in direct communication with the pubic symphysis joint space. Vague periarticular marrow edema-like signal and enhancement at the pubic symphysis was thought to be infectious/inflammatory in etiology (Figure-2).

Figure 1 - Axial MRI.

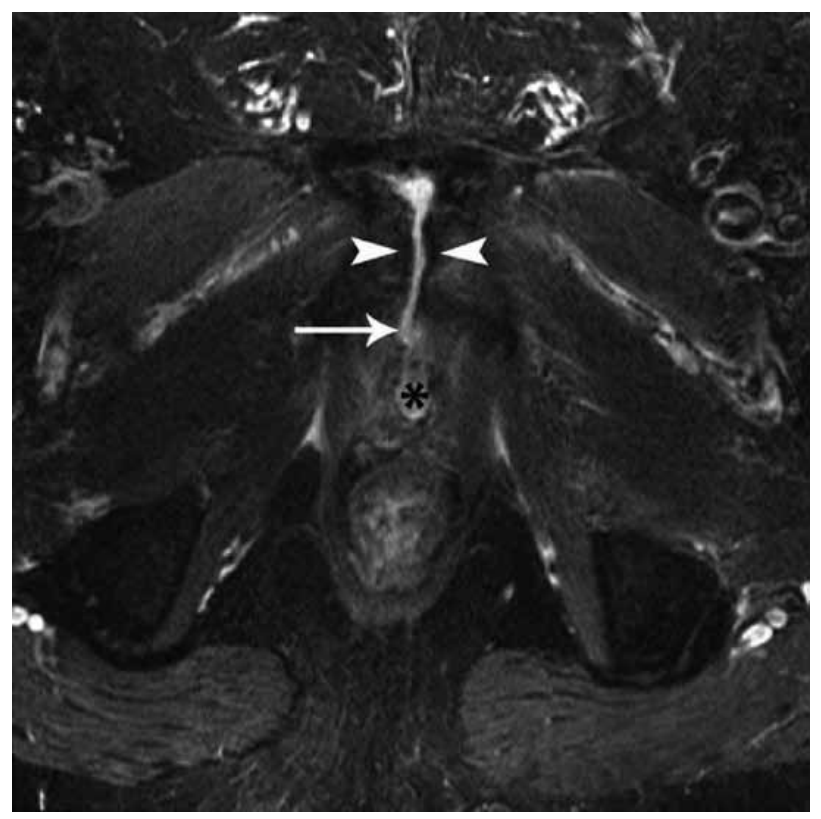

Axial image through the inferior pelvis demonstrates tract-like high signal intensity fistulous communication (white arrow) between the bladder base (black asterisk) and pubic symphysis (white arrowheads).

Similar inflammatory changes were also evident in the proximal adductor musculature bilaterally. The patient's symptoms resolved after one month of urethral catheter drainage, antibiotics, and anti-inflammatory therapy. A confirmatory CT cystogram before and after voiding demonstrated the absence of persistent fistula prior to catheter removal.

\section{DISCUSSION}

The role of MRI in the workup of a fistula involving the urinary tract is evolving. While limited by the same patient requirements as other MR exams, looking for urinary tract fistulae via MRI offers the advantage of being relatively less invasive than retrograde studies and offers excellent imaging of soft tissues, which can provide evidence for fistula even when the tract itself is not visualized. The ability to image inflammatory changes in soft tissues is especially helpful in a situation where external physical findings are not present or indeterminate. This is especially true in the case above where a symptomatic

Figure 2 - Coronal MRI.

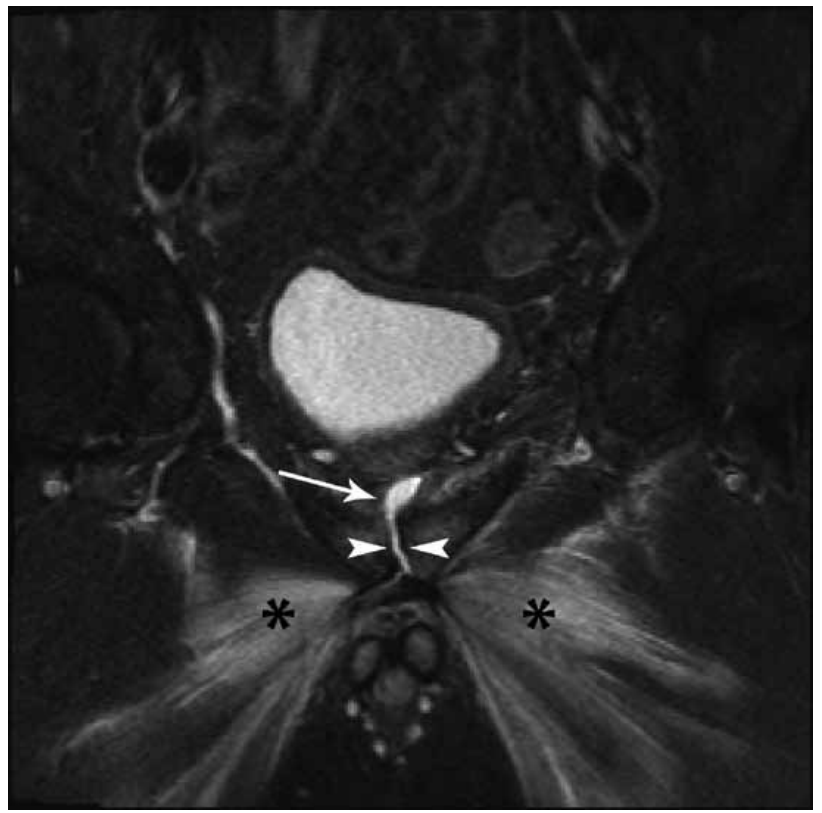

Coronal image through the anterior pelvis demonstrates fluid signal within the pubic symphysis (white arrowheads) in continuity with fistulous tract (white arrow). Vague marrow signal abnormality involving the adjacent pubic bones with concomitant broad areas of edema-like signal tracking along the adductor musculature bilaterally (black asterisks). Signal abnormity in the pubic bones and adductor musculature is likely inflammatory in etiology.

patient had a negative CT scan of the pelvis while MRI demonstrated significant pathology. Continued progress in the field of MR imaging provides yet another useful imaging adjunct in the diagnosis and management of these challenging Urologic problems (3).

\section{LIST OF ABBREVIATIONS}

$\mathrm{CT}=$ computed tomography

MRI = magnetic resonance imaging

$\mathrm{MR}=$ magnetic resonance 


\section{REFERENCES}

1. Beer E. Periostitis of the symphysis and descending rami of the pubis following suprapubic operations. Int J Med Surg 1924 37: 224.

2. Kats E, Venema PL, Kropman RF: A rare complication after endoscopic resection of the prostate: osteitis pubis due to a prostate-symphysis fistula. J Urol. 1997; 157: 624.

\section{ARTICLE INFO}

Int Braz J Urol. 2013; 39: 288-90

Submitted for publication:

January 05, 2013

Accepted after revision:

February 18, 2013
3. Semelka RC, Hricak H, Kim B, Forstner R, Bis KG, Ascher SM, et al.: Pelvic fistulas: appearances on MR images. Abdom Imaging. 1997; 22: 91-5.

Correspondence address:

Dr. David D. Thiel

Mayo Clinic

Department of Urology

Davis Building $3 \mathrm{E}$

4500 San Pablo Rd.

Jacksonville, FL 32224, USA

Fax: +1 904 953-2218

E-mail: thiel.david@mayo.edu 